\title{
ANALYZING THE DENSITY OF RESIDENTS IN SHANGHAI TO EXAMINE THE ROLE OF BIG DATA IN THE DEVELOPMENT OF SMART CITIES
}

\begin{tabular}{|c|c|c|c|}
\hline \multicolumn{4}{|c|}{$\begin{array}{l}\text { Rishi Sikka } \\
\text { ersity, Mathura, Uttar Pradesh, India }\end{array}$} \\
\hline Journal & \multicolumn{3}{|c|}{$\begin{array}{l}\text { Samvakti Journal of Research in Information Technology } \\
\text { https://www.sjrit.samvaktijournals.com } \\
\text { Volume } 2 \text { Year of Volume } 2021 \text { Page No : } 8 \text { - } 15\end{array}$} \\
\hline Discipline & \multicolumn{3}{|l|}{ Big Data } \\
\hline Conference & \multicolumn{3}{|c|}{$\begin{array}{l}\text { A virtual international conference on redefining and transforming the role of higher } \\
\text { education in sustainable development }\end{array}$} \\
\hline Conference & \multicolumn{3}{|c|}{ Start Date: September 30, 2021} \\
\hline Dates & \multirow{2}{*}{\multicolumn{3}{|c|}{$\begin{array}{l}\text { End Date : September 30, } 2021 \\
\text { JAIN (Deemed-to-be University) in association with Council for Industrial } \\
\text { Innovation and Research }\end{array}$}} \\
\hline Institute Name & & & \\
\hline $\begin{array}{l}\text { Date Received } \\
\text { ID } \\
\text { Dol }\end{array}$ & $\begin{array}{l}\text { : November } 19,2021 \\
: 2021.02 .11 \\
: 10.46402 / 2021.02 .11\end{array}$ & $\begin{array}{l}\text { Publication Date } \\
\text { Paper Type } \\
\text { Dol URL }\end{array}$ & $\begin{array}{l}\text { : December 10, } 2021 \\
\text { : Conference Paper } \\
\text { : https://dx.doi.org/10.46402/2021.02.11 }\end{array}$ \\
\hline
\end{tabular}

\section{ABSTRACT}

A significant amount of study has been done in recent decades to evaluate data from social networks based on the region in order to emphasize their applicability. This social networking data based on geography may be used to create models and predictions. methods for analyzing and reproducing spatiotemporal patterns and user activity symmetry, as well as volume estimates. Distinct density estimation methods are used in the present research to examine the number of times people checking in in a certain period of time depth using a database of social networks depending on region obtained from Sina-Weibo, commonly known as Weibo, during a particular time time in ten distinct Shanghai areas, China. The goal of this research is to look at the density of users in Shanghai based on Weibo geolocation data and compare it to other cities using univariate and bivariate thickness estimate methods, such as point density and kernel density estimation (KDE). The study's main findings include: I geographical aspects of users' behavior, such as check-in-based activity centers, (ii) the practicality of employing check- 
in data to describe the interaction between users and social networks, and (iii) the presentation of unambiguous findings for regulatory or governing authorities in urban planning. The present research demonstrates that point density and kernel density estimates may be used together. The KDE techniques may be used to model spatial patterns utilizing geospatial datasets. Finally, we may infer that using the KDE method, we can analyze individual check-in behavior as well as larger trends in the general public for the establishment of smart cities. The goal of this article is to identify denser areas so that authorities may split people's mobility along the same routes or, at the very least, keep the situation under control and avoid any additional annoyance.

KEYWORDS: Data Analysis, KDE, Point Density, Smart City Architecture, Spatial Analysis.

\section{INTRODUCTION}

In the modern era, a big quantity of individual people data from social networks depending on geolocation such as Fb, Twitter, Snapchat, and Weibo data is available because of the rise in helpfulness of intelligent gadgets, can give data on individual behaviour as well as geography and various demographic data and mentality such as internet actions, phone calls, texts, and more. Scientists have been encouraged to investigate a wide range of subjects in sequence to grow accurate estimates for classifying the temporal and spatial allocation of individual people and the populace overall, taking into account individuals as a source of data due to their moves and use of LBSNs via a few computing phone that records the day ${ }^{[1]}$. By accumulating such data about users' knowledge, we can analyze it in conjunction combines historical, economic, and geographic variables, allowing us to see patterns like differences in people's sleeping habits around the world, or how individuals from other country spends their summers and winter holidays, and so forth. The computation of the thickness of opinions surrounding depending on every outputting lipid membrane characteristics is known as point density ${ }^{[2]}$.

A region is created everywhere every tissues center, with all of the opinions inside the city totalled and split by the neighborhood's zone. The radius is said to have no effect on the density value computation. Despite the fact that the quantity of vertices in a large neighborhood grows, the density is measured by distributing the quantity of points by the area, which grows comparatively ${ }^{[3]}$. Despite being a clear and easy approach, the points density function does not provide any data about the spatiotemporal arrangement inside the channel capacity ${ }^{[4]}$. By increasing the numbers of points in a vast region, a greater diameter may be used to compute a more generalized output. Kernel density estimation $(K D E)$ is a well-known method for analyzing latitudinal opinion patterns. KDE with spatially adjustable bandwidths is favored ended KDE through absurd bandwidths in many situations. However, determining bandwidth for adaptable KDE is very twisted expensive, 
especially for large-sample point pattern analysis ${ }^{[4]}$. The the implementation of adaptable $\mathrm{KDE}$ to huge opinion information circles, that is frequent in current large statistics age, is hampered by this computational difficulty. In this article, we delve into the geographical features of check-in data and expand its use.

In addition, the accompanying investigation questions will be addressed in this study: the primary Depending on the user check-ins, the hub of activity, and (ii) if it is possible to explain the connection among social media and users usage using LBSN data. We also used density estimation maps to show the impact of multivariate thickness besides multivariate thickness (KDE). To display the users' check-in habits, we utilized To analyze Weibo data, use Dense and KDE. We looked at several elements of LBSN statistics to look at separate activities shows the thickness of check-ins in Shanghai ended period. We also looked at Check-in behaviour in 10 Shanghai areas, according to LBSN data: Pudong New Zone, Changning, Baoshan, Edition, Definition is offered, Hongkou, Putuo, Yangpu, Corrective, and Xuhui. In the context of this study, check-in behavior refers to how users interrelate through LBSN and engage in events such as distribution their position by uploading a geolocated photo or remark. The main cause these ten districts were chosen is because they are all linked to the city core ${ }^{[5]}$. For our empirical investigation, we utilized a dataset from Weibo, which is one of China's the most widely used social media platforms Among our contributions are a comparative of opinion thickness besides KDE use to discoveries from the geo-place record, as well as the checked-in thickness of operators for a illustration of the overall open in Shanghai. This research may help in a variety of disciplines, including urban functions and environmental impacts, Sustainability, growth, and crisis response in cities are all important factors to consider. depending on crowd densities inside the city, as well as future research in these areas. This work is completed as part of the master's thesis ${ }^{[6]}$. The popularity of social media has grown in tandem with the widespread Cell telephones and the Web have become more popular. expanded people's capacity to travel to new locations across the globe.

Emails, texts twitter and other means of communications are becoming more popular. often enabled by social network apps, allowing people all over the globe to communicate. Users can communicate geo-location-related information in the form of textual, audio, and video with the advancement of mobile gadget technologies, and with the Cellphones have been more popular in previous years, a significant revolution in geo-positioning capabilities has occurred, inspiring workers to use locality-built services (LBSs), resulting LBSs are becoming more popular and commercialized. Among the first research on LBSN use looked at why and how individuals utilize them. presents an empirical study of LBSNs, whereas presents a research into the spatiotemporal properties of LBSNs. With the innovation and accessibility of portable devices, monitoring a user's location has grown 
simpler in recent years ${ }^{[7]}$. A dataset that included 100,000 users' data over a six-month period. For every call made via a mobile phone, the dataset included the position of the user's nearest base station tower, enabling researchers to get estimates information and position of every client during a particular time period used this data to perform a KDE analysis in order to make work routines more predictable. Researchers discovered that being able It's simple to control one's identity by sharing info across thousands of LBSN members., meet new people, and do new activities. looked into methods for anticipating user transitions in the future. Furthermore, sophisticated methods for prediction in LBSNs, for example, utilized It's simple to control one's identify by sharing info across thousands of LBSN members. illnesses, potentially paving the way for novel real-time epidemiological calculations. Foursquare check-in data to identify small spatial clusters in urban regions, which may be useful in Planned economies, allocation of resources, and city design growth. In the past, point density and kernel density estimates were employed for many reasons.

Peter Beskow and Paul Evangelista proposed a concentration of spatial points technique for precisely and meaningfully understanding spatial point activity density Using Point Density Analysis, Chao et al. investigated the geographical China's archeological location dispersal. The extensive material on the Point Density function. investigated the the use of feature densities in conjunction with Euclidian range to improve grape juice formulation. Utilized the KDE method to perform fast pattern analysis of geographic large data. Through the use of KDE on different illnesses, the a variable set by the user of KDE, which affects the resolution of mappin ${ }^{[7]}$. Lichman and Smyth conducted an investigation on the information's typical sparseness and variability of 3-3- D movement designs used $\mathrm{KDE}$. To investigate health inequalities and other public health problems. Utilized includes the density of the points and the density of the kernel. With selection biased data, the authors utilized Kernel density estimations. KDE has been used to do a great deal of work.

Previous studies in Weibo data density estimation Applied a sole approach, as an example KDE, to calculate thickness on charts aimed at a specific objective, including this kind of gender perspective in verdant parks, as well as concentrated on open areas, tourist, and center of interest suggestions, among other things. Researchers utilized Weibo to investigate the geographical features In Wuhan, researchers used a single approach to analyze check-in data: spatial study of registration data using point density., and Keep a look behaviour depending on genders KDE and also KDE had remained used to choose bandwidth and intensity parameters that are appropriate for local circumstances, as well as to measure spatial segregation. The KDE method has been used to represent geo-location data spatially, giving a more generic methodology for assessing spatial density that is both adaptable and robust ${ }^{[8]}$. 


\section{Study Area and Database:}

Shanghai is located within the Yangtze River Delta's eastern and western borders. 30400-31530 N and 12052-122120 E. Shanghai has an area of $8359 \mathrm{~km} 2$ and had a gross domestic product (GDP) of In 2018, 480 million dollars (USD) were spent. In 2016, Shanghai was split There are 16 semi in all, comprising one municipality and 15 districts. The ten linked Shanghai districts are examined for this research. Puxi is home to Changning, Huangpu, Putuo, Hongkou, Xuhui, Jingan, and Yangpu. These 7 districts are together known as Shanghai's downtown region or city-center. The data for our research came "Weibo" is a China weblog. This system is focused on region. focuses on sharing a user's present position Geo-spatial variables are true variables. locations that the user specifies. Checking in and connecting with people in the network are how users to connect to the program., much like any other LBSN ${ }^{[9]}$. Weibo, one of China's most significant LBSNs, experienced an exponential increase in activity and awareness shortly after its debut on August 14,2009, and has now matured. We chose Weibo data since it is not only China's biggest LBSN, but it also includes sophisticated geodata of many choices and methods a variety of social features that attract users to check-in often. Weibo reported in December 2018 that it has there are more than 500 billion authorized customers. regularly utilizing the site in 2018, with 462 million regular vigorous consumers. In 2018, the most recent official estimate of daily active users was 200 million. As a result, in order to investigate user activity patterns, we must focus on users that use the app on a regular basis ${ }^{[10]}$.

The information gathered via the usage of LBSN apps is subject to significant privacy issues and limitations. In China, it is very difficult to get open and trustworthy geo-locationbased data. For this research, the LBSN dataset was collected from Weibo between January and April of this year. Weibo provides an accessible geodatabase which may be accessed using the Weibo API., which is written in Python. The dataset includes characteristics such the user's ID, the date, and the hour, as well as geo-places, groups, and venue terms, thanks to Weibo's open geodatabase. Not at all private info is available due to the users' desire for concealment. As a result, check-in data depicts users' designs and behaviours of regular activities, as well as the typical person's daily life activities. Since there are so many verification and engaged customers, Shanghai was selected as the research location. The business applications interface (API) was used during January to March of 2016. gathered 824,304 check-ins from 11,108 individuals inside the Shanghai administrative limits (API). Noises, wildcard entries, and incorrect records were removed from the Weibo data. To address the problem of heterogeneity and the importance of the dataset, the following criteria were used for data preparation and cleaning: it's possible Due to the problem of heterogeneity, only active users should be included in the sample of users in order to guarantee a high degree of generalisability. 
From January to March 2016, the collection of records utilized in our research comprises the customer ID, the customer's latitudes, and the customer's longitudinal, which includes 10,317 legitimate users and 786,650 check-ins. The research is being carried out in Shanghai, China's financial capital.

\section{DISCUSSION}

The number of visitors was estimated using as a proxy, geotagged social media network check-in data. This method is both time and labor-intensive, and it provides excellent geographical coverage. Users of LBSs share information on their interests and interests, as well as whatever they provide, why people discuss it, and with whomever they discuss it, thanks to the fusion of technology that had previously been separate fueled the growth of LBSNs. Weibo data, as the study demonstrates, is a useful tool for evaluating urban functioning and studying spatiotemporal variables. Because we can gather appropriate and big-scale information around a whole town in extra depth using social media data to assess user behavior, the best source for geographical information analytics is Weibo data. KDE is a program. that balances occasion based on their distances and two required parameters. The bandwidth, or control distance, is the first of them. The bandwidth you choose has a significant impact on performance. The premium purpose $\mathrm{K}$, which is usually a regular occupation, is the second parameter. The kernel's bandwidth is a unrestricted variable that has a significant effect on the final estimation. The volume is regular, and there are no anomalies. a mean of 0 and a variance of 1 . In the limit $\mathrm{h} 0$ (no smoothing), when the estimation is the result of adding $\mathrm{n}$ delta operations centered on the classifies of the studied data record, an extreme scenario occurs.

The lack of data has been the most significant stumbling block for LBSN research, owing to concerns about physical security and privacy. With LBSNs' capacity to disclose users' and their friends' current geo-locations, there are serious worries regarding users' privacy. Individual users' privacy is a concern, However, it also applies to institutions. or corporate users that share info in LBSNs. Secret information can occasionally disclosed willingly or inadvertently. While data may occasionally be collected by providing users with incentives and advantages in exchange for their info, the position of a customer could be determined via LBSN amenities such as Wechat Nearby. Some LBSN services also include capabilities that allow users to locate their friends. Based on the information given above, it can be inferred that Shanghai's city center has the greatest check-in density. Furthermore, because of the ease of access to transportation amenities, density is greater around roads and subways. The findings are consistent with reality, since it is clear that the city center and regions linked by the subway, as well as certain other tourist and educational institutions outside of the city center, are the most populated locations in any large city. To the best of our intellect, This is the initial investigation. in Shanghai to use 


\section{Development of Smart Cities}

Weibo data to evaluate denser locations utilizing KDE and Point density estimates based on check-in behavior. The identification of more packed and busy locations in our research may help authorities enhance urban planning, crowd management at big events, and offer relevant information to users on when to visit a particular location, among other things.

\section{CONCLUSION}

The use of LBSNs to analyze activity patterns is beneficial since they provide data of different modalities linked to every customer. To far, this data has been utilized and studied for a variety of reasons, including activity and location recommendations and neighborhood characterization. In this research, we looked at check-in trends depending on users' geo-information and location. We discovered several intriguing trends (for example, weak densities near the city's boundaries and high densities in the city center) that generally correspond to and are consistent with real-world predictions.

Our data has the benefit of reflecting the general behavior of a large number of people with a variety of backgrounds. We looked at the distribution of users' check-ins in ten distinct Shanghai districts, emphasizing various features of geo-referenced data. We used point density to depict the number of users in Shanghai and specific areas. We used kernel density on the same research region and dataset to display our results in two dimensions. We also utilized regression models to determine the dataset's relevance. This research may be useful in detecting congested locations in Shanghai so that regulatory or management authorities can better monitor and assist such regions, particularly during festivals, public events, catastrophes, and urban development. However, by comparing and contrasting two distinct methods, point density and KDE, we are able to not only compare but also verify our findings. This research may be helpful in the creation and upkeep of a smart city. 


\section{REFERENCES}

[1] N. L. Bragazzi, H. Dai, G. Damiani, M. Behzadifar, M. Martini, and J. Wu, "How big data and artificial intelligence can help better manage the covid-19 pandemic," Int. J. Environ. Res. Public Health, 2020, doi: 10.3390/ijerph17093176.

[2] I. Abaker et al., "International Journal of Information Management The role of big data in smart city," Int. J. Inf. Manage., 2016.

[3] R. Dwevedi, V. Krishna, and A. Kumar, "Environment and big data: Role in smart cities of India," Resources, 2018, doi: 10.3390/resources7040064.

[4] "Role of Big Data and Analytics in Smart Cities," Int. J. Sci. Res., 2016, doi: 10.21275/v5i2.nov161007.

[5] Y. Pan, Y. Tian, X. Liu, D. Gu, and G. Hua, "Urban Big Data and the Development of City Intelligence," Engineering. 2016, doi: 10.1016/J.ENG.2016.02.003.

[6] T. Hong, C. Chen, J. Huang, N. Lu, L. Xie, and H. Zareipour, "Guest Editorial Big Data Analytics for Grid Modernization," IEEE Transactions on Smart Grid. 2016, doi: 10.1109/TSG.2016.2593358.

[7] M. Maksimovic, "The role of green internet of things (G-loT) and big data in making cities smarter, safer and more sustainable," Int. J. Comput. Digit. Syst., 2017, doi: 10.12785/IJCDS/060403.

[8] P. C. Kiran, "When Smart Cities Meet Big Data.," Voice Data, 2016.

[9] Y. LI and Y. YANG, "Toward the Smart Eco-City Governance: The Vital Role of Big Data," DEStech Trans. Environ. Energy Earth Sci., 2017, doi: 10.12783/dteees/eccsd2016/5817.

[10] S. M. Willems et al., "The potential use of big data in oncology," Oral Oncology. 2019, doi: 10.1016/j.oraloncology.2019.09.003. 